\title{
ELEMENTS OF BUILT-IN DIAGNOSTICS FOR RESONANCE DRIVE WITH ADAPTIVE CONTROL SYSTEM
}

\author{
Akinfiev T.*, Armada M.** \\ * IMASH RAS, Russia. Current address: IAI CSIC, Carretera de \\ Camporreal, Km. 0,200, La Poveda, 28500 Arganda del Rey, Madrid, \\ Spain.E-mail: teodor@iai.csic.es \\ ** IAI CSIC, Carretera de Camporreal, Km. 0,200, La Poveda, 28500 \\ Arganda del Rey, Madrid, Spain.E-mail: armada@iai.csic.es
}

\begin{abstract}
Resonance drives are considered with adaptive control for robotics. The use of these drives allows increasing machine's quickness several times and decreasing energy expenses simultaneously 10-50 times. Elements of artificial intellect are introduced into the drives. It has been shown that micro-controller used for adaptive control, can be also used for getting a diagnostic information on a condition of drive's components during work process. Algorithms are presented for evaluation of condition of resilient members, reduction gear, etc.
\end{abstract}

Keywords: Start-stop movement, resonance drive, adaptive control, built-in diagnostic, robotics.

\section{INTRODUCTION}

In this report resonance drives (RDs) [1] are considered for machines, which working elements makes episodic movements with stops and stoppages (MSS machines). It has been shown previously [4] that the use of $\mathrm{RDs}$ in robotics allows increasing robot's quickness substantially and decreasing energy expenses simultaneously. It has been shown also that to provide reliable operation of RDs, these drives have to have adaptive control system, which usually is made on microchip basis. Such control system makes analysis of movement parameters and outputs corresponding control actions on a drive motor.

Moreover, the same information on movement parameters of a drive used by control system could be also used for monitoring of technical condition of drive's components. It is important to note that in many cases to make a monitoring there is no need to put on additional sensors or switch on a special regime of robot's operation. Special algorithms have been elaborated of getting a diagnostic information immediately during work operation. Particularly, it is possible to get operative information on springs' condition, their tuning, friction forces, clearances in kinematic circuit etc.

Results of analytical calculations, which allow calculating the values of constructive parameters of RDs on the basis of analysis of dynamic properties of RDs, are discussed in the report also. Results are presented of simulating with the use of MATLABSIMULINK package. Results are given of full-scale experiments with different RDs.

\section{THE SIMPLEST CONSTRUCTION OF RESONANCE DRIVE}

It is well known from the theory of fluctuations that acceleration and braking in resonance oscillatory systems are performed at the expense of passive resilient members, while motor serves only for making up for friction losses in the system. The idea of using resonance shed new light on the problem of increasing of speed of MSS machines. The resonance effect allows, in comparison with conventional drives, the enhancement of performance quickness of machine, and, at the same time, the reduction of power input. This is done without a complication of the construction of machine on the whole. A special technique of designing has allowed creating [4] various MSS machines with resonance properties: Resonance Drives (RD).

The simplest type of RD is intended for progressive (Fig. 1) or revolving (Fig. 2) movement of mobile link 1 with stop and fixation in extreme positions. Resilient members 2 between carriage and support structure are adjusted so that mobile link should be in a state of balance when it is at the centre of a distance between extreme positions. 


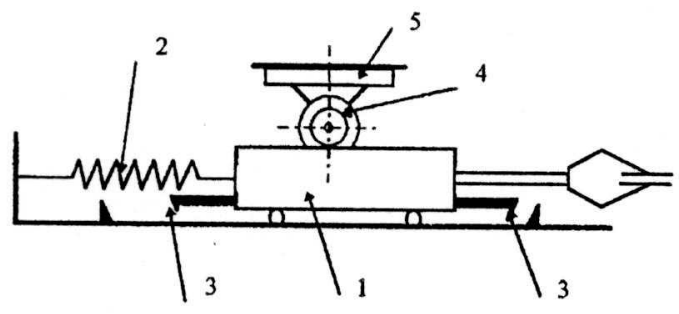

Figure 1. Simplest RD for progressive movement.

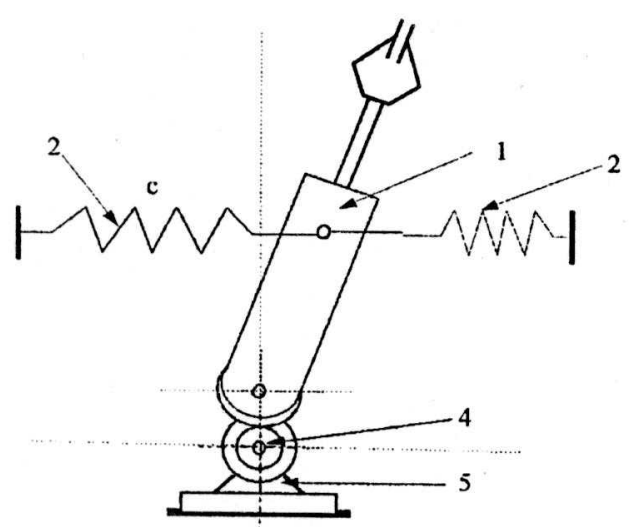

Figure 2. Simplest RD for revolving movement.

When mobile link is in one or another extreme, retaining devices 3 automatically retains it. In the initial moment mobile link is always in one or another extreme. To start a motion of mobile link from initial position, a movable part of retaining device has to be disengaged from stationary part. With mobile link released, a resilient member moves it towards its target position by accelerating it over the first half of the trajectory and decelerating it over the second half of the trajectory. When mobile link arrives in the target position, retaining device automatically retains it again. Drive motor is connected with mobile link by means of reduction gear 4 and serves only for compensation for friction losses.

It has to be mentioned that the simplest RD possesses a substantial limitation - it allows to stop a mobile link only in extreme positions. However, other kinematic circuits of RD have been elaborated, which allow bringing mobile link to a stop in any intermediate position. They allow also moving from every position to any other one. Usually, non-linear resilient members are used in such drives. Moreover, it is possible to use RD even in the case when a magnitude of mobile link's displacement is not known beforehand. Particularly, when walking robot's leg makes upright movement, way's profile determines magnitude of step and that does not interfere using special RD for such movement. We have to note that RD does not have to have a fixing rod necessarily. Different RDs of substantially simple construction without fixing rods have been elaborated.

Irrespective of constructive characteristics of any $\mathrm{RD}$, the main idea remains unaltered - drive motor is used for compensation of losses in a system and inertia forces are compensated at the expense of passive resilient elements. Usually, this allows increasing quickness several times in comparison with conventional drives, while energy expenses decrease 10-50 times.

For example, resonance robot MARS-3 (Fig. 3), which has been produced in small series, has three degrees of freedom. Load capacity of this robot is $0,5 \mathrm{~kg}$, speed of load's displacement is up to $2 \mathrm{~m} / \mathrm{s}$ with electric motor's power of only $4 \mathrm{~W}$ [4].

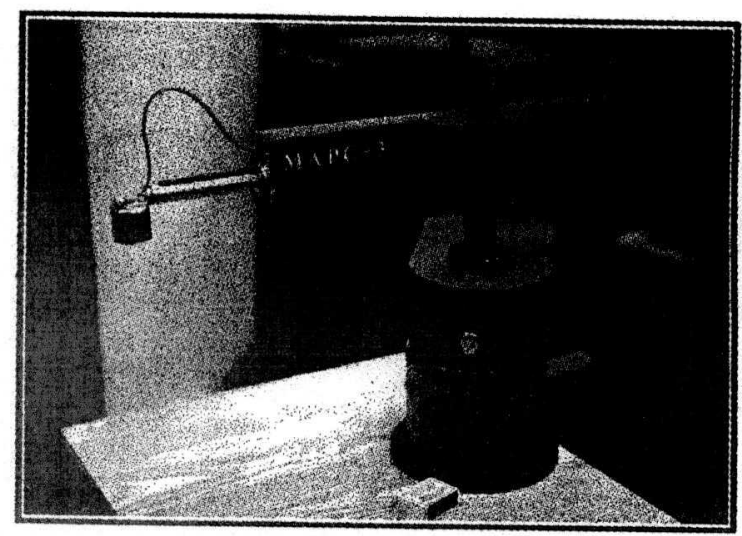

Figure 3. Resonance robot MARS-3.

Resonance stepper motor (Fig. 4) with 60W power turns 90 degrees a load of $32 \mathrm{~kg}$ in $0,5 \mathrm{~s}$ [4].

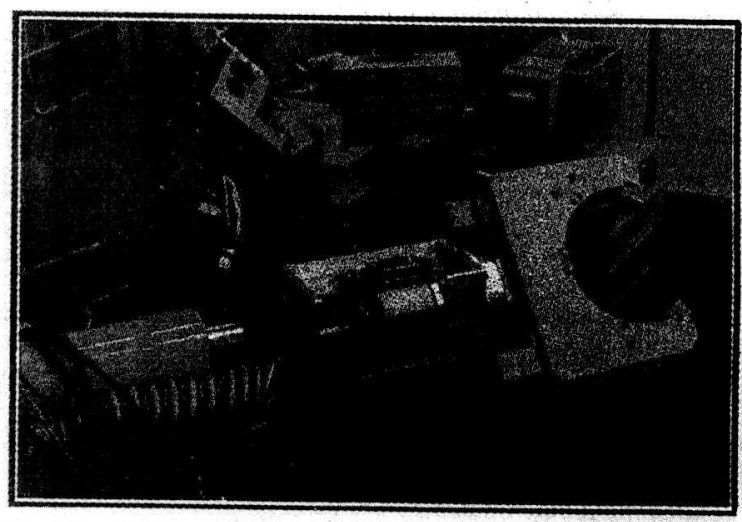

Figure 4. Resonance stepper motor.

Resonance portal robot (Fig. 5) has three degrees of freedom. Load capacity of this robot is $10 \mathrm{~kg}$, speed of displacement of a load is up to $1.5 \mathrm{~m} / \mathrm{s}$ with electric motor's power of 90W [4]. 


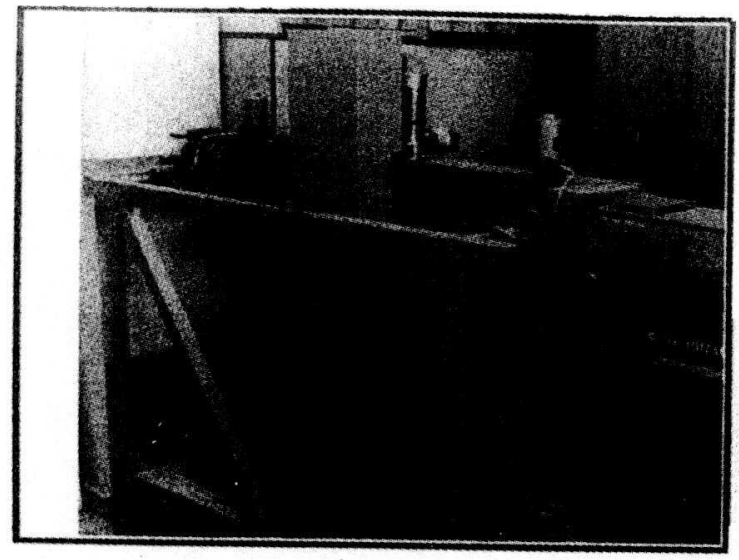

Figure 5. Resonance portal robot.

It has been also shown experimentally [5] that the use of $\mathrm{RD}$ could increase walking robot' speed 10 times.

\section{CONTROL SYSTEM}

Important feature of RD is that it has two independent energy resources. One of them is a passive resilient member, another is a drive motor. Drive motor's power is many times less than that of resilient element. For maintenance of reliable work of $R D$ it is necessary to make it available an effective interaction between these two resources of energy. This problem becomes complicated because of character of movement, which is formed, on the whole, by passive resilient member and depends substantially on mass (moment of inertia) of moving part. So, when using RD in robotics, character of movement can change substantially from one movement to another because in some cases robot moves without payload, and in other cases - with payloads of different mass. In fact, there is no information about movement's law before beginning of the movement. Under such conditions, control system has to apply such voltage to drive motor, which would compensate energy losses during the movement. Let's note that even friction forces are not known beforehand; they could be different during different movements both because of casual cause and because of payload's influence.

Nevertheless, for such conditions a universal algorithm of adaptive control has been elaborated that makes it available a reliable work of $\mathrm{RD}$ [3]. It is based on the use of idea of movement' simmetrization. That means that if on the first part of trajectory a movement begins with zero speed, then symmetric (relative to middle position) movement would end with zero speed also at the end position. Thus, it would mean that during movement a drive motor would transfer to a mobile link some energy, which would be equal to energy losses during this movement. The algorithm uses the idea of selftraining [6], but unlike conventional use of self- training algorithms, training is made immediately during work process on the part of trajectory of movement. Such approach allowed getting universal adaptive control system, i.e. such control system, which could be used for any RD.

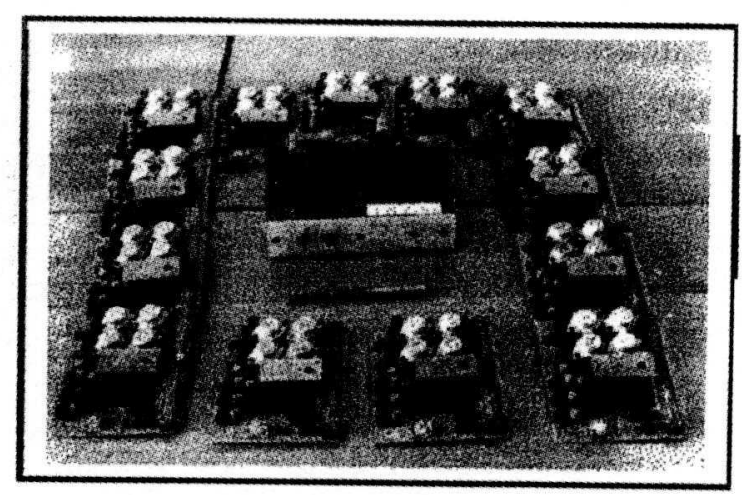

Figure 6. Control systems.

For implementation of adaptive control system to be possible, RD has to have a sensor of co-ordinate and/or of speed. Then a special microprocessor (Fig.6) would be able to make records of parameters of movement on the first part of trajectory. On the second part of trajectory this microprocessor would feed drive motor with a voltage that would minimize the difference between real speed and the speed on hypothetical symmetric curve.

\section{BUILD-IN DIAGNOSTICS}

It has been shown $[4,5]$ that $\mathrm{RD}$ with adaptive control has to have sensors and micro-controller, in which a processing of data is made, on parameters of drive's movement. The same micro-controller could be used for getting diagnostic information on condition of components of drive. Let us note that a major portion of this information could be get immediately during the process of work operations with the help of special processing of data on movement's parameters.

\subsection{Diagnostics of springs' condition.}

Spring is one of the most important components of RD. Many end users had worried about spring being substantially extended or even broken with time. It has to be noted, however, that plastic deformation of springs leads to substantial change of their rigidity long before their destruction begins. That is why a continual control of springs' rigidity is so important.

It is well known from oscillation theory that friction forces have a negligible effect on period of oscillations in resonance system. When using linear springs, period of oscillations depends only on rigidity of spring and on mass of mobile part. Taking into consideration that character of movement of RD 
corresponds to movement of suitable simulating oscillation system [1], for a case of linear spring we have:

$$
c=\frac{\pi^{2} m}{t^{2}}
$$

where $m-$ is a mass of mobile link, $c$ - is a rigidity of resilient member, $t-$ is a time of displacement of mobile link from one position to another.

Thus, a time of displacement of mobile link from one extreme position to another (with fixed mass of this link) characterizes rigidity of resilient member. Availability of microprocessor in control system allows calculating a magnitude of rigidity after each idle run of drive (i.e. such movement when a drive is moving without a payload). However, there is no need to know the exact magnitude of spring's rigidity in practice. It is quite sufficient to have a routine information about how this rigidity is changing with time in comparison with the initial rigidity. It means that at the moment of the initial start up of a drive it is necessary to record the magnitude of time of drive's idle run into microprocessor's memory. During a work process, one has to make a comparison of this magnitude with actual time's magnitude of every idle run of a drive. If actual idle run time's magnitude falls outside the before established limits, then microprocessor would be able to generate a special signal on necessity of reconditioning of the drive.

When using non-linear springs, amplitude of oscillations (i.e. magnitude of run of mobile link) produces an effect on a period of oscillations also. So, in this case it is necessary to measure a time of movement only for the runs, which fall in the same distance with the run recorded as reference run.

Let us note that the results of numerous experimental researches of RDs $[4,5]$ have shown that properly calculated springs operate with a high degree of reliability.

\subsection{Diagnostics of losses in drive.}

It has been noted above that under proper tuning RD motor serves only for compensation of the losses during movement. It is supposed that the balance position of mobile link is in the middle of a distance between extreme positions. Moreover, it is supposed that a situation is considered when a movement takes place without a payload. Under such conditions it is possible to determine losses inside a system by calculating a value of energy transferred from motor to mobile link. Generally speaking, it is not a possibility of calculating the exact value of losses that counts but a possibility to note significant change in this value. So, it is enough to have a possibility to calculate to within a constant factor the energy transferred from motor to mobile link.

In the easiest case, when electromagnet constant of electro-motor is small in comparison with electromechanical constant, to calculate the instant value of power transferred from motor to mobile link one can use the formula [7]:

$$
W=i U-i^{2} R \text {, }
$$

where $i-$ is a current in motor armature, $U$ - is a voltage applied to motor, $R-$ is a resistance of motor armature.

The value of armature resistance is known beforehand (the known motor parameters). The information on voltage applied to the motor in each moment is contained in micro-controller that carries out drive's control. So, it is necessary to have only a special sensor of magnitude of current and to bring a signal from this sensor into micro-controller.

Taking into consideration a discrete character of control, micro-controller can calculate the energy transferred by the drive to mobile link during the next movement using the formula:

$$
E=\sum_{k=1}^{N} \tau_{k}\left(i_{k} U_{k}-i_{k}^{2} R\right),
$$

where $k$-is a number of time interval, $\tau_{k}$ - is a . value of time interval.

During initial start up we can record a magnitude of $E^{*}$ into micro-controller's memory, and during each idle run a record could be also made of a current magnitude of $E$. If error between $E$ and $E^{*}$ would exceed a set up limit, the system could give a special signal on the necessity of lubrication of bearings.

\subsection{Diagnostics of resilient members' tuning.}

It has been mentioned above that the balance position of mobile link on a resilient member has to be in the middle of a distance between extreme positions. It is clear that a small deviation in springs' tuning would not destroy RD's normal operation. Compensation would be made automatically by adaptive control system, which would provide a transfer of necessary amount of energy to mobile link. However, taking into consideration limited possibilities of motors used in $\mathrm{RD}$, it is advantageous to make resilient members' tuning close to optimal.

Usually, drive's tuning is made during maintenance. It seems to be expedient to have information at that moment on springs' tuning. To have it, it is necessary to make two movements of the drive - one in positive and another in negative directions. During both these movements one has to calculate energy transferred from motor to resilient member due to the method described in 4.2. If the difference between these two values would not exceed the before established value, then it means that springs are tuned properly. If the difference is big, it is necessary to adjust the springs. Adjustments are made by moving a position of fastening of one spring. So, the balance position of mobile link would move in the direction, where mobile link has been 
moving when it has been getting more energy from control system.

It seems to be advantageous to have a special algorithm in drive control system, which would make episodic automatic check of springs' tuning (for example, once a day at the initial switches on of the drive). To make this, it is sufficient to make only two movements in opposite directions and to compare energy values transferred from motor to mobile link. If the difference between these two values is not big, drive is ready to operate. If the difference is considerably big, a tuning is necessary.

Let's note that when it is necessary to switch off a drive for a rather long time, it make sense not to leave the drive in one of the extreme positions (with one of resilient members in a highly deformed state). It is much better to leave it in the middle position with both resilient members equally deformed. In this case resilient members' tuning would not change even if the drive would not operate for a long time.

\subsection{Diagnostics of condition of reduction gear.}

It is specific for $\mathrm{RD}$ that there are fixing rods that hold mobile link in positioning position. Fixing rods could be of two types. One type prevents mobile link's movements both in positive and negative directions. Such fixing rods are used in RDs with springs that have "negative" rigidity [2]. Another type of fixing rods prevents movements only in one direction (Fig. 1). In this case resilient members pin mobile link against fixing rod with a high force, which prevents this link's movements in opposite direction even with motor switched on. So, when using fixing rods of both types, motor's switching on in any direction does not lead to a movement of mobile link (if fixing rod is switched on). That allows evaluating clearances easily in motor mobile link kinematic circuit.

It is supposed that mobile link is in one of positioning positions and is fixed by fixing rod. When there is a clearance in motor - mobile link kinematic circuit, a motor can be in a random position within limits of this clearance. At that moment drive's motor has to be fed with a voltage of some polarity, and after that - with a voltage of opposite polarity. That leads to motor's first making a turn into one direction within the limits of the clearance of kinematic circuit (magnitude of movement is of a random character). Then the motor makes such a turn into opposite direction, which corresponds to exact value of clearance in kinematic circuit. A signal (corresponding to angle of motor's turn) from motor's built-in encoder comes to microcontroller and is compared with analogous signal that had been recorded into micro-controller at the initial start up of RD. If the difference between these values falls outside the established limits, then micro-controller gives a signal on a necessity of a check of reduction gear. It makes sense to check reduction gear episodically, for example, once a day at the initial switching on of a drive.

During such check there is no need to feed drive motor with nominal voltage; usually, it is sufficient to feed drive motor with a voltage corresponding to $25-30 \%$ of nominal voltage.

In some cases it is possible to check also a condition of the motor simultaneously. To make that, it is sufficient to record into micro-controller a magnitude of motor's current immediately before motor's switching off (locked motor's current under some fixed voltage). If this magnitude would be much lower than corresponding value recorded at the initial start up of RD, then that means a contact unit in a motor does not work well. If current's magnitude is substantially higher than previously recorded magnitude, that means that in the motor itself there was a shorting of a few turns of winding. In both cases micro-controller has to give a signal on necessity of operator's intervention.

\section{Acknowledgments}

The authors would like to acknowledge the received support from EC ESPRIT - INCO Commission, from Consejo Superior de Investigaciones Cientificas (CSIC, Spain) and from Academy of Sciences of Russia.

\section{REFERENCES}

[1] T. Akinfiev. "Resonance Manipulation Systems with Electric Drives". USSR Academy of Sciences, Mashinovedeniye, \#6, pp. 18-23. 1983.

[2] T. Akinfiev, V. Dauge, A. Romanov. "The Resonance Drive". USSR Patent \#1357219. 1986.

[3] T. Akinfiev. "Method of Controlling Mechanical Resonance Hand", United States Patent \$4958113. 1990.

[4] T. Akinfiev. "The Resonance Drives with Adaptive Control". Proceedings of the 11th ASCE Engineering Mechanics Conference, Ft. Lauderdale, Florida, USA, 1996. New York: ASCE, 0-78440172-1, pp. 947-950. 1996.

[5] T. Akinfiev, M. Armada. "Resonance and QuasiResonance Drives for Start-Stop Regime". Proceedings of the 6th International Conference MECHATRONICS'98, Skovde, Sweden. 1998. Pergamon, pp. 91-96, 1998.

[6] V. Besekerskii, E. Popov. Theory of Automatic Control Systems. Nauka, Moscow. 1975.

[7] M. Chilikin, A. Sandler. General Course of Electric Drives. Energoizdat, Moscow. 1981. 